\title{
Profil d'une publication: la Revue internationale d'Education pour la Santé
}

\author{
Annette Kaplun ', Genève
}

La Revue internationale d'Education pour la Santé a vingt ans! En 1957, juste à cette même époque de l'année, le premier numéro était en préparation. La naissance d'une revue est toujours un événement, même si celle-ci n'a que de modestes ambitions. Mais nos ambitions étaient-elles si modestes? Dans la notice annonçant le lancement de la Revue, on demandait aux lecteurs: «Désirez-vous être tenu au courant des progrès de l'éducation sanitaire dans votre pays et dans le monde? Désirez-vous savoir comment d'autres personnes, dans d'autres pays, s'attaquent aux problèmes de l'éducation sanitaire du public? Désirezvous des informations sur les méthodes nouvelles et les recherches en matière d'éducation sanitaire?" Puis on annonçait le but de la Revue: servir de tribune internationale pour l'échange d'expériences en même temps que d'instrument pratique de travail pour tous ceux qui sont à l'avant-garde de l'éducation de la santé dans le monde.

Aujourd'hui, ces buts sont restés les mêmes, avec, toutefois, une place plus grande accordée à la recherche. Cette tendance reflète le souci des travailleurs de la santé d'aborder les problèmes éducatifs de la façon la plus scientifique possible, en se basant sur des données foumies par l'analyse d'expériences soigneusement planifiées.

La RIES n'est pas une revue tout à fait «comme les autres». Et cela à divers titres. C'est une enfant dont les parents sont très occupés et qui a un peu grandi toute seule - vigoureusement, d'ailleurs, car elle est lue aujourd'hui à peu près dans tous les pays du monde et son bureau de rédaction n'a jamais besoin de se préoccuper d'obtenir des articles: ils lui parviennent spontanément des quatre coins du monde, nombreux, trop nombreux même pour sa capacité de publication. Mais, en fait, qui sont ces «parents»? Son titre, «organe officiel de l'Union internationale d'Education pour la Santé» cache une réalité rédactionnelle peutêtre inattendue: en effet, l'équipe responsable - une mini-équipe dont le siège est à Genève - est composée de bénévoles, ce qui laisse deviner à la fois l'enthousiasme et la conviction qui animent ses membres aussi bien que les soirées tardives consacrées à la préparation de la Revue. Mentionnons également le rôle largement «bénévole» de la maison d'arts graphiques qui en assume la publication et l'originalité du profil se précise. Ajoutons enfin que les membres du comité de lecture anglais et français ont leur siège dans les capitales de deux continents.

Voici donc le cadre particulier de réflexion et d'action qui préside à la vie de la RIES. Au cours des vingt

'Directrice de la Revue internationale d'Education pour la Santé, 3, rue Viollier, CH-1207 Genève, Suisse. années de son existence, ce sont au total plus de 11000 pages qui ont été publiées par la RIES, reflétant les réflexions, les expériences et les recherches de près de 1000 auteurs venant d'horizons variés: santé publique, sciences sociales, éducation, écologie, etc. $\mathrm{Au}$ début, la Revue paraissait en français et en anglais. Puis des éditions en langues espagnole et allemande sont venues étendre son rayonnement. Aujourd'hui, une formule multilingue a été adoptée, comportant des articles publiés dans leur langue originale, avec des résumés dans les autres langues. Cette édition est soutenue par le Comité français d'Education pour la Santé, d'une part, et le Centre fédéral allemand d'Education pour la Santé, d'autre part. De plus, des suppléments réservés à des articles de plus longue haleine paraissent avec les éditions anglaise et allemande.

Peu à peu, la RIES a été entraînée à trouver un autre canal pour la diffusion des nombreuses études et expériences qui lui étaient soumises. C'est ainsi que plusieurs séries d'ouvrages techniques ont vu le jour. Il y a, tout d'abord, naturellement, la série des actes des conférences internationales de l'Union, qui contiennent une mine d'informations précieuses: L'homme dans son milieu (Philadelphie, 1962); Communication et modification du comportement (Buenos Aires, 1969); Vingt années d'éducation pour la santé: bilan et prospective (Paris, 1973); et enfin, Education sanitaire et politique de santé dans la dynamique du développement (Ottawa, 1976).

Une autre série de publications a été entreprise avec le Centre fédéral allemand d'Education pour la Santé. II s'agit d'ouvrages portant sur la nutrition, les maladies cardio-vasculaires, la méthodologie d'éducation pour la santé, la thérapie du comportement en relation avec l'usage du tabac et, enfin, l'éducation pour la santé en Europe.

D'autres livres ont été publiés en collaboration avec la Fédération mondiale des associations de santé publique sur la planification familiale et l'environnement, et un autre avec le Centre d'Education pour la Santé en Angleterre, sur les sciences du comportement dans le domaine de la santé et de la maladie.

Mais le «best-seller» demeure le CHECK du Professeur Turner, adapté en français sous le titre de Mémento. Ce petit ouvrage est en effet extrêmement utile à toute personne soucieuse d'améliorer le rendement de son travail; il contient non seulement d'utiles informations sur les principes de base qui président à l'intervention éducative, sur la communication, la dynamique de l'action au niveau des collectivités, et l'usage des mass media, mais aussi des réflexions pleines de sagesse sur les relations humaines. 